\title{
Testing stability in a spatial unilateral autoregressive model
}

\author{
SÁndor Baran ${ }^{1}$, Gyula PAP ${ }^{2}$ And Kinga Sikolya ${ }^{1}$ \\ ${ }^{1}$ Faculty of Informatics, University of Debrecen \\ Kassai út 26, H-4028 Debrecen, Hungary \\ ${ }^{2}$ Bolyai Institute, University of Szeged \\ Aradi vértanúk tere 1, H-6720 Szeged, Hungary
}

\begin{abstract}
Least squares estimator of the stability parameter $\varrho:=|\alpha|+|\beta|$ for a spatial unilateral autoregressive process $X_{k, \ell}=\alpha X_{k-1, \ell}+\beta X_{k, \ell-1}+\varepsilon_{k, \ell}$ is investigated. Asymptotic normality with a scaling factor $n^{5 / 4}$ is shown in the unstable case, i.e., when $\varrho=1$, in contrast to the $\operatorname{AR}(\mathrm{p})$ model $X_{k}=\alpha_{1} X_{k-1}+\cdots+\alpha_{p} X_{k-p}+\varepsilon_{k}$, where the least squares estimator of the stability parameter $\varrho:=\alpha_{1}+\cdots+\alpha_{p}$ is not asymptotically normal in the unstable, i.e., in the unit root case.
\end{abstract}

Key words: unstable spatial unilateral autoregressive process; unit root tests.

2010 Mathematics Subject Classifications: primary 62M10; secondary 62F12.

\section{Introduction}

Consider a spatial unilateral autoregressive process $\left\{X_{k, \ell}: k, \ell \in \mathbb{Z}, k+\ell \geq 0\right\}$ defined by

$$
X_{k, \ell}= \begin{cases}\alpha X_{k-1, \ell}+\beta X_{k, \ell-1}+\varepsilon_{k, \ell}, & \text { for } k+\ell \geq 1 \\ 0, & \text { for } k+\ell=0\end{cases}
$$

where $\left\{\varepsilon_{k, \ell}: k, \ell \in \mathbb{Z}, k+\ell \geq 1\right\}$ are independent random variables with $\mathrm{E}\left(\varepsilon_{k, \ell}\right)=0$ and $\operatorname{Var}\left(\varepsilon_{k, \ell}\right)=1$. This model is stable in case of $|\alpha|+|\beta|<1$ and unstable if $|\alpha|+|\beta|=1$ (see Whittle (1954); Besag (1972); Basu and Reinsel (1993)), hence $\varrho:=|\alpha|+|\beta|$ can be considered as a stability parameter.

For a set $H \subset\left\{(k, \ell) \in \mathbb{Z}^{2}: k+\ell \geq 1\right\}$, the least squares estimator (LSE) $\left(\widehat{\alpha}_{H}, \widehat{\beta}_{H}\right)$ of the coefficients $(\alpha, \beta)$ based on the observations $\left\{X_{k, \ell}:(k, \ell) \in H\right\}$ can be obtained by 
minimizing the sum of squares

$$
\sum_{(k, \ell) \in H}\left(X_{k, \ell}-\alpha X_{k-1, \ell}-\beta X_{k, \ell-1}\right)^{2}
$$

with respect to $\alpha$ and $\beta$, and it has the form

$$
\left[\begin{array}{c}
\widehat{\alpha}_{H}^{*} \\
\widehat{\beta}_{H}^{*}
\end{array}\right]=\left(A_{H}^{*}\right)^{-1} b_{H}^{*}, \quad \text { where } \quad A_{H}^{*}:=\sum_{(k, \ell) \in H}\left[\begin{array}{c}
X_{k-1, \ell} \\
X_{k, \ell-1}
\end{array}\right]\left[\begin{array}{c}
X_{k-1, \ell} \\
X_{k, \ell-1}
\end{array}\right]^{\top}, \quad b_{H}^{*}:=\sum_{(k, \ell) \in H} X_{k, \ell}\left[\begin{array}{c}
X_{k-1, \ell} \\
X_{k, \ell-1}
\end{array}\right] \text {. }
$$

Model (1.1) has been investigated in details by several authors. Paulauskas (2007) determined the exact asymptotic behaviour of the variances of the process, while Baran et al. (2007) proved the asymptotic normality of the LSE of the coefficients $(\alpha, \beta)$ both in stable and unstable cases.

The limiting behavior of the LSE of the stability parameter $\varrho$ has not been treated yet, but such a stability parameter is well investigated in case of unstable $\operatorname{AR}(p)$ processes, see the unit root tests, e.g., in Hamilton (1994, Section 17, Table 17.3, Case 1). Namely, for the simplicity, in case of an $\operatorname{AR}(1)$ process $Y_{k}=\varrho Y_{k-1}+\zeta_{k}, k \in \mathbb{N}$, with $Y_{0}:=0$ and an i.i.d. sequence $\left\{\zeta_{k}: k \in \mathbb{N}\right\}$ having mean 0 and positive variance, the LSE of the parameter $\varrho$ based on a sample $\left\{Y_{1}, \ldots, Y_{n}\right\}$ takes the form

$$
\widehat{\varrho}_{n}=\frac{\sum_{k=1}^{n} Y_{k-1} Y_{k}}{\sum_{k=1}^{n} Y_{k}^{2}}, \quad n \in \mathbb{N},
$$

see, e.g., Hamilton (1994, 17.4.2), and, by Hamilton $(1994,17.4 .7)$, in the unstable case, i.e., when $\varrho=1$,

$$
n\left(\widehat{\varrho}_{n}-1\right) \stackrel{\mathcal{D}}{\longrightarrow} \frac{\int_{0}^{1} \mathcal{W}_{t} \mathrm{~d} \mathcal{W}_{t}}{\int_{0}^{1} \mathcal{W}_{t}^{2} \mathrm{~d} t} \quad \text { as } n \rightarrow \infty
$$

where $\left(\mathcal{W}_{t}\right)_{t>0}$ is a standard Wiener process. Here $n\left(\widehat{\varrho}_{n}-1\right)$ is called Dickey-Fuller unit root statistics. It turns out that in case of unstable spatial unilateral autoregressive processes asymptotic normality holds, see Theorem 1.1.

With the help of the stability parameter $\varrho$ the model can also be written in the form

$$
X_{k, \ell}= \begin{cases}\alpha\left(X_{k-1, \ell}-\operatorname{sign}(\alpha \beta) X_{k, \ell-1}\right)+\varrho \operatorname{sign}(\beta) X_{k, \ell-1}+\varepsilon_{k, \ell}, & \text { for } k+\ell \geq 1, \\ 0, & \text { for } k+\ell=0\end{cases}
$$

This reparametrization can be called the canonical form of Sims et al. (1990) (see also Hamilton $(1994,17.7 .6))$. Observe that (1.2) gives four different models according to the signs of $\alpha$ and $\beta$. Hence, in order to derive estimators of the parameters $(\alpha, \varrho)$ one should have information about these signs.

For a set $H \subset\left\{(k, \ell) \in \mathbb{Z}^{2}: k+\ell \geq 1\right\}$, the least squares estimator $\left(\widehat{\alpha}_{H}, \widehat{\varrho}_{H}\right)$ of $(\alpha, \varrho)$ based on the observations $\left\{X_{k, \ell}:(k, \ell) \in H\right\}$ can be obtained by minimizing the sum of squares

$$
\sum_{(k, \ell) \in H}\left[X_{k, \ell}-\alpha\left(X_{k-1, \ell}-\operatorname{sign}(\alpha \beta) X_{k, \ell-1}\right)-\varrho \operatorname{sign}(\beta) X_{k, \ell-1}\right]^{2}
$$


with respect to $\alpha$ and $\varrho$, and it has the form

$$
\left[\begin{array}{c}
\widehat{\alpha}_{H} \\
\widehat{\varrho}_{H}
\end{array}\right]=A_{H}^{-1} b_{H}
$$

where

$$
\begin{aligned}
A_{H} & :=\sum_{(k, \ell) \in H}\left[\begin{array}{c}
X_{k-1, \ell}-\operatorname{sign}(\alpha \beta) X_{k, \ell-1} \\
\operatorname{sign}(\beta) X_{k, \ell-1}
\end{array}\right]\left[\begin{array}{c}
X_{k-1, \ell}-\operatorname{sign}(\alpha \beta) X_{k, \ell-1} \\
\operatorname{sign}(\beta) X_{k, \ell-1}
\end{array}\right]=B A_{H}^{*} B^{\top}, \\
b_{H} & :=\sum_{(k, \ell) \in H} X_{k, \ell}\left[\begin{array}{c}
X_{k-1, \ell}-\operatorname{sign}(\alpha \beta) X_{k, \ell-1} \\
\operatorname{sign}(\beta) X_{k, \ell-1}
\end{array}\right]=B b_{H}^{*}, \quad \text { with } \quad B:=\left[\begin{array}{cc}
1 & -\operatorname{sign}(\alpha \beta) \\
0 & \operatorname{sign}(\beta)
\end{array}\right] .
\end{aligned}
$$

Obviously, this estimator is well defined if $\alpha \beta \neq 0$ and then we have

$$
\left[\begin{array}{c}
\widehat{\alpha}_{H} \\
\widehat{\varrho}_{H}
\end{array}\right]=\left(B^{\top}\right)^{-1}\left[\begin{array}{c}
\widehat{\alpha}_{H}^{*} \\
\widehat{\beta}_{H}^{*}
\end{array}\right] \text {. }
$$

Now, let us define an estimator of $\beta$ by $\widehat{\beta}_{H}:=\left(\widehat{\varrho}_{H}-\operatorname{sign}(\alpha) \widehat{\alpha}_{H}\right) \operatorname{sign}(\beta)$. Short calculation shows that $\widehat{\alpha}_{H}=\widehat{\alpha}_{H}^{*}$ and $\widehat{\beta}_{H}=\widehat{\beta}_{H}^{*}$.

For $k, \ell \in \mathbb{Z}$ with $k+\ell \geq 1$, consider the triangle

$$
T_{k, \ell}:=\left\{(i, j) \in \mathbb{Z}^{2}: i+j \geq 1, i \leq k \text { and } j \leq \ell\right\}
$$

For simplicity, we shall write $T_{n}:=T_{n, n}$ for $n \in \mathbb{N}$.

Theorem 1.1 Let $\left\{\varepsilon_{k, \ell}: k, \ell \in \mathbb{Z}, k+\ell \geq 1\right\}$ be independent random variables with $\mathrm{E}\left(\varepsilon_{k, \ell}\right)=0, \operatorname{Var}\left(\varepsilon_{k, \ell}\right)=1$ and $\sup \left\{\mathrm{E}\left(\varepsilon_{k, \ell}^{4}\right): k, \ell \in \mathbb{Z}, k+\ell \geq 1\right\}<\infty$.

If $|\alpha|+|\beta|<1$ and $\alpha \beta \neq 0$ then

$$
n\left(\widehat{\varrho}_{T_{n}}-1\right) \stackrel{\mathcal{D}}{\longrightarrow} \mathcal{N}\left(0,\left(1+\kappa_{\alpha, \beta} \operatorname{sign}(\alpha \beta)\right)^{-1} \sigma_{\alpha, \beta}^{-2}\right)
$$

and

$$
\left[\begin{array}{l}
n\left(\widehat{\alpha}_{T_{n}}-\alpha\right) \\
n\left(\widehat{\beta}_{T_{n}}-\beta\right)
\end{array}\right] \stackrel{\mathcal{D}}{\longrightarrow} \mathcal{N}\left(\left[\begin{array}{l}
0 \\
0
\end{array}\right], \frac{1}{2 \sigma_{\alpha, \beta}^{2}\left(1-\kappa_{\alpha, \beta}^{2}\right)}\left[\begin{array}{cc}
1 & -\kappa_{\alpha, \beta} \\
-\kappa_{\alpha, \beta} & 1
\end{array}\right]\right),
$$

as $n \rightarrow \infty$, where

$$
\begin{aligned}
& \sigma_{\alpha, \beta}^{2}:=((1+\alpha+\beta)(1+\alpha-\beta)(1-\alpha+\beta)(1-\alpha-\beta))^{-1 / 2}, \\
& \kappa_{\alpha, \beta}:=\frac{\left(1-\alpha^{2}-\beta^{2}\right) \sigma_{\alpha, \beta}^{2}-1}{2 \alpha \beta \sigma_{\alpha, \beta}^{2}} . \\
& \text { If }|\alpha|+|\beta|=1 \text { and } \alpha \beta \neq 0 \text { then } \\
& n^{5 / 4}\left(\widehat{\varrho}_{T_{n}}-1\right) \stackrel{\mathcal{D}}{\longrightarrow} \mathcal{N}\left(0, \psi_{\alpha}\right)
\end{aligned}
$$


and

$$
\left[\begin{array}{l}
n\left(\widehat{\alpha}_{T_{n}}-\alpha\right) \\
n\left(\widehat{\beta}_{T_{n}}-\beta\right)
\end{array}\right] \stackrel{\mathcal{D}}{\longrightarrow} \mathcal{N}\left(\left[\begin{array}{l}
0 \\
0
\end{array}\right], \varphi_{\alpha}\left[\begin{array}{cc}
1 & -\operatorname{sign}(\alpha \beta) \\
-\operatorname{sign}(\alpha \beta) & 1
\end{array}\right]\right),
$$

as $n \rightarrow \infty$, where

$$
\varphi_{\alpha}:=\frac{|\alpha|(1-|\alpha|)}{2} \quad \text { and } \quad \psi_{\alpha}:=\frac{15 \sqrt{\pi|\alpha|(1-|\alpha|)}}{2^{9 / 2}} .
$$

We remark that (1.3) is a direct consequence of (1.4) which is the first statement of Theorem 1.1 of Baran et al. (2007). Further, (1.6) has already proved in Baran et al. (2007), too, but with a far more complicated method than here.

Now, observe that to prove the statement of Theorem 1.1 in the unstable case it suffices to show

$$
\left[\begin{array}{c}
n\left(\widehat{\alpha}_{T_{n}}-\alpha\right) \\
n^{5 / 4}\left(\widehat{\varrho}_{T_{n}}-1\right)
\end{array}\right] \stackrel{\mathcal{D}}{\longrightarrow} \mathcal{N}\left(0, \Sigma_{\alpha}\right) \quad \text { as } n \rightarrow \infty
$$

where

$$
\Sigma_{\alpha}:=\left[\begin{array}{cc}
\varphi_{\alpha} & 0 \\
0 & \psi_{\alpha}
\end{array}\right]
$$

Limit (1.5) is obvious, but (1.7) also implies $n\left(\widehat{\varrho}_{T_{n}}-1\right) \stackrel{\mathrm{P}}{\longrightarrow} 0$. In this way (1.6) follows from (1.7) and

$$
\begin{aligned}
{\left[\begin{array}{l}
n\left(\widehat{\alpha}_{T_{n}}-\alpha\right) \\
n\left(\widehat{\beta}_{T_{n}}-\beta\right)
\end{array}\right] } & =\left[\begin{array}{c}
n\left(\widehat{\alpha}_{T_{n}}-\alpha\right) \\
\operatorname{sign}(\beta)\left(n\left(\widehat{\varrho}_{T_{n}}-1\right)-\operatorname{sign}(\alpha) n\left(\widehat{\alpha}_{T_{n}}-\alpha\right)\right)
\end{array}\right] \\
& =n\left(\widehat{\alpha}_{T_{n}}-\alpha\right)\left[\begin{array}{c}
1 \\
-\operatorname{sign}(\alpha \beta)
\end{array}\right]+n\left(\widehat{\varrho}_{T_{n}}-1\right)\left[\begin{array}{c}
0 \\
\operatorname{sign}(\beta)
\end{array}\right] .
\end{aligned}
$$

Further, we can write

$$
\left[\begin{array}{c}
\widehat{\alpha}_{T_{n}}-\alpha \\
\widehat{\varrho}_{T_{n}}-1
\end{array}\right]=A_{T_{n}}^{-1} d_{T_{n}}
$$

where

$$
d_{T_{n}}:=\sum_{(k, \ell) \in T_{n}} \varepsilon_{k, \ell}\left[\begin{array}{c}
X_{k-1, \ell}-\operatorname{sign}(\alpha \beta) X_{k, \ell-1} \\
\operatorname{sign}(\beta) X_{k, \ell-1}
\end{array}\right]
$$

and by the continuous mapping theorem (1.7) is a consequence of the convergence

$$
\left(\widetilde{A}_{T_{n}}, \widetilde{d}_{T_{n}}\right):=\left(\left[\begin{array}{cc}
n^{-1} & 0 \\
0 & n^{-5 / 4}
\end{array}\right] A_{T_{n}}\left[\begin{array}{cc}
n^{-1} & 0 \\
0 & n^{-5 / 4}
\end{array}\right],\left[\begin{array}{cc}
n^{-1} & 0 \\
0 & n^{-5 / 4}
\end{array}\right] d_{T_{n}}\right) \stackrel{\mathcal{D}}{\longrightarrow}(\widetilde{A}, \widetilde{d})
$$


as

$n \rightarrow \infty$, where

$$
\widetilde{A}:=\left[\begin{array}{cc}
1 / \varphi_{\alpha} & 0 \\
0 & 1 / \psi_{\alpha}
\end{array}\right] \quad \text { and } \quad \widetilde{d} \stackrel{\mathcal{D}}{=} \mathcal{N}(0, \widetilde{A})
$$

Obviously, (1.8) can be verified by proving the following two propositions.

Proposition 1.2 Under the conditions of Theorem 1.1

$$
\widetilde{A}_{T_{n}} \stackrel{\mathrm{P}}{\longrightarrow} \widetilde{A} \quad \text { as } n \rightarrow \infty .
$$

Proposition 1.3 Under the conditions of Theorem 1.1

$$
\widetilde{d}_{T_{n}} \stackrel{\mathcal{D}}{\longrightarrow} \mathcal{N}(0, \widetilde{A}) \quad \text { as } n \rightarrow \infty
$$

The aim of the following discussion is to show that it suffices to prove Propositions 1.2 and 1.3 for $\alpha>0$ and $\beta>0$ implying $\varrho=\alpha+\beta$. In this case we have

$$
\begin{aligned}
& \widetilde{A}_{T_{n}}=\sum_{(k, \ell) \in T_{n}}\left[\begin{array}{cc}
n^{-2}\left(X_{k-1, \ell}-X_{k, \ell-1}\right)^{2} & n^{-9 / 4}\left(X_{k-1, \ell}-X_{k, \ell-1}\right) X_{k, \ell-1} \\
n^{-9 / 4}\left(X_{k-1, \ell}-X_{k, \ell-1}\right) X_{k, \ell-1} & n^{-5 / 2} X_{k, \ell-1}^{2}
\end{array}\right], \\
& \widetilde{d}_{T_{n}}=\sum_{(k, \ell) \in T_{n}}\left[\begin{array}{c}
n^{-1}\left(X_{k-1, \ell}-X_{k, \ell-1}\right) \varepsilon_{k, \ell} \\
n^{-5 / 4} X_{k, \ell-1} \varepsilon_{k, \ell}
\end{array}\right] .
\end{aligned}
$$

Model equation (1.1) implies that random variable $X_{k, \ell}$ can be expressed as a linear combination of the variables $\left\{\varepsilon_{i, j}:(i, j) \in T_{k, \ell}\right\}$, namely,

$$
X_{k, \ell}=\sum_{(i, j) \in T_{k, \ell}}\left(\begin{array}{c}
k+\ell-i-j \\
k-i
\end{array}\right) \alpha^{k-i} \beta^{\ell-j} \varepsilon_{i, j}
$$

for $k, \ell \in \mathbb{Z}$ with $k+\ell \geq 1$. If $\alpha+\beta=1$ we can also write

$$
X_{k, \ell}=\sum_{(i, j) \in T_{k, \ell}} \mathrm{P}\left(S_{k+\ell-i-j}^{(\alpha)}=k-i\right) \varepsilon_{i, j}
$$

where $S_{n}^{(\alpha)}$ is a binomial random variable with parameters $(n, \alpha)$.

Let $\alpha<0, \beta<0$ implying $\varrho=-\alpha-\beta$ and put $\varepsilon_{k, \ell}^{*}:=(-1)^{k+\ell} \varepsilon_{k, \ell}$ for $k, \ell \in \mathbb{Z}$ with $k+\ell \geq 1$. Then $\left\{\varepsilon_{k, \ell}^{*}: k, \ell \in \mathbb{Z}, k+\ell \geq 1\right\}$ are independent random variables with $\mathrm{E}\left(\varepsilon_{k, \ell}^{*}\right)=0$, and $\operatorname{Var}\left(\varepsilon_{k, \ell}^{*}\right)=1$. Consider the zero start triangular spatial AR process $\left\{X_{k, \ell}^{*}: k, \ell \in \mathbb{Z}, k+\ell \geq 0\right\}$ defined by

$$
X_{k, \ell}^{*}= \begin{cases}-\alpha X_{k-1, \ell}^{*}-\beta X_{k, \ell-1}^{*}+\varepsilon_{k, \ell}^{*}, & \text { for } k+\ell \geq 1 \\ 0, & \text { for } k+\ell=0\end{cases}
$$


In this case (1.2) takes the form

$$
X_{k, \ell}^{*}= \begin{cases}-\alpha\left(X_{k-1, \ell}^{*}-X_{k, \ell-1}^{*}\right)+\varrho X_{k, \ell-1}^{*}+\varepsilon_{k, \ell}^{*}, & \text { for } k+\ell \geq 1, \\ 0, & \text { for } k+\ell=0 .\end{cases}
$$

Then, by representation $(1.12)$,

$$
X_{k, \ell}^{*}=\sum_{(i, j) \in T_{k, \ell}}\left(\begin{array}{c}
k+\ell-i-j \\
k-i
\end{array}\right)(-\alpha)^{k-i}(-\beta)^{\ell-j} \varepsilon_{i, j}^{*}=(-1)^{k+\ell} X_{k, \ell}
$$

for $k, \ell \in \mathbb{Z}$ with $k+\ell \geq 0$. Hence,

$$
\begin{aligned}
A_{T_{n}}^{*} & =\sum_{(k, \ell) \in T_{n}}\left[\begin{array}{cc}
n^{-2}\left(X_{k-1, \ell}^{*}-X_{k, \ell-1}^{*}\right)^{2} & -n^{-9 / 4}\left(X_{k-1, \ell}^{*}-X_{k, \ell-1}^{*}\right) X_{k, \ell-1}^{*} \\
-n^{-9 / 4}\left(X_{k-1, \ell}^{*}-X_{k, \ell-1}^{*}\right) X_{k, \ell-1}^{*} & n^{-5 / 2}\left(X_{k, \ell-1}^{*}\right)^{2}
\end{array}\right] \\
= & {\left[\begin{array}{cc}
-1 & 1 \\
1 & -1
\end{array}\right] \widetilde{A}_{T_{n}}\left[\begin{array}{cc}
-1 & 1 \\
1 & -1
\end{array}\right], } \\
d_{T_{n}}^{*} & =\sum_{(k, \ell) \in T_{n}}\left[\begin{array}{c}
-n^{-1}\left(X_{k-1, \ell}^{*}-X_{k, \ell-1}^{*}\right) \varepsilon_{k, \ell}^{*} \\
n^{-5 / 4} X_{k, \ell-1}^{*} \varepsilon_{k, \ell}^{*}
\end{array}\right]=\left[\begin{array}{cc}
-1 & 1 \\
1 & -1
\end{array}\right] \widetilde{d}_{T_{n}},
\end{aligned}
$$

where $\widetilde{A}_{T_{n}}$ and $\widetilde{d}_{T_{n}}$ have forms (1.10) and (1.11), respectively. Consequently, in order to prove Propositions 1.2 and 1.3 for $\alpha<0$ and $\beta<0$ it suffices to prove them for $\alpha>0$ and $\beta>0$.

Next, let $\alpha<0, \beta>0$ implying $\varrho=-\alpha+\beta$ and put $\varepsilon_{k, \ell}^{+}:=(-1)^{k} \varepsilon_{k, \ell}$ for $k, \ell \in \mathbb{Z}$ with $k+\ell \geq 1$. Then $\left\{\varepsilon_{k, \ell}^{+}: k, \ell \in \mathbb{Z}, k+\ell \geq 1\right\}$ are again independent random variables with $\mathrm{E}\left(\varepsilon_{k, \ell}^{+}\right)=0$, and $\operatorname{Var}\left(\varepsilon_{k, \ell}^{+}\right)=1$. Consider the zero start triangular spatial AR process $\left\{X_{k, \ell}^{+}: k, \ell \in \mathbb{Z}, k+\ell \geq 0\right\}$ defined by

$$
X_{k, \ell}^{+}= \begin{cases}-\alpha X_{k-1, \ell}^{+}+\beta X_{k, \ell-1}^{+}+\varepsilon_{k, \ell}^{+}, & \text {for } k+\ell \geq 1 \\ 0, & \text { for } k+\ell=0 .\end{cases}
$$

Now $X_{k, \ell}^{+}=(-1)^{k} X_{k, \ell}$ and (1.2) takes the form

$$
X_{k, \ell}^{+}= \begin{cases}-\alpha\left(X_{k-1, \ell}^{+}-X_{k, \ell-1}^{+}\right)+\varrho X_{k, \ell-1}^{+}+\varepsilon_{k, \ell}^{+}, & \text {for } k+\ell \geq 1, \\ 0, & \text { for } k+\ell=0\end{cases}
$$

and

$$
\begin{aligned}
A_{T_{n}}^{+} & =\sum_{(k, \ell) \in T_{n}}\left[\begin{array}{cc}
n^{-2}\left(X_{k-1, \ell}^{+}+X_{k, \ell-1}^{+}\right)^{2} & -n^{-9 / 4}\left(X_{k-1, \ell}^{+}+X_{k, \ell-1}^{+}\right) X_{k, \ell-1}^{+} \\
-n^{-9 / 4}\left(X_{k-1, \ell}^{+}+X_{k, \ell-1}^{+}\right) X_{k, \ell-1}^{+} & n^{-5 / 2}\left(X_{k, \ell-1}^{+}\right)^{2}
\end{array}\right] \\
& =\left[\begin{array}{cc}
-1 & 1 \\
1 & -1
\end{array}\right] \widetilde{A}_{T_{n}}\left[\begin{array}{cc}
-1 & 1 \\
1 & -1
\end{array}\right], \\
d_{T_{n}}^{+} & =\sum_{(k, \ell) \in T_{n}}\left[\begin{array}{c}
-n^{-1}\left(X_{k-1, \ell}^{+}+X_{k, \ell-1}^{+}\right) \varepsilon_{k, \ell}^{+} \\
n^{-5 / 4} X_{k, \ell-1}^{+} \varepsilon_{k, \ell}^{+}
\end{array}\right]=\left[\begin{array}{cc}
-1 & 1 \\
1 & -1
\end{array}\right] \widetilde{d}_{T_{n}},
\end{aligned}
$$


where $\widetilde{A}_{T_{n}}$ and $\widetilde{d}_{T_{n}}$ have forms (1.10) and (1.11), respectively.

In the same way one can handle the case $\alpha>0, \beta<0$ implying $\varrho=\alpha-\beta$ by considering $\left\{X_{k, \ell}^{\circ}: k, \ell \in \mathbb{Z}, k+\ell \geq 0\right\}$ defined by

$$
X_{k, \ell}^{\circ}= \begin{cases}\alpha X_{k-1, \ell}^{\circ}-\beta X_{k, \ell-1}^{\circ}+\varepsilon_{k, \ell}^{\circ}, & \text { for } k+\ell \geq 1 \\ 0, & \text { for } k+\ell=0\end{cases}
$$

with $\varepsilon_{k, \ell}^{\circ}=(-1)^{\ell} \varepsilon_{k, \ell}$.

\section{Results on the covariance structure}

In order to prove Propositions 1.2 and 1.3 one has to know the asymptotic behaviour of the covariances of the process $X_{k, \ell}$. By representation (1.12) we obtain that for all $k_{1}, \ell_{1}, k_{2}, \ell_{2} \in$ $\mathbb{Z}$ with $k_{1}+\ell_{1} \geq 0$ and $k_{2}+\ell_{2} \geq 0$, and for all $\alpha, \beta \in \mathbb{R}$,

$$
\operatorname{Cov}\left(X_{k_{1}, \ell_{1}}, X_{k_{2}, \ell_{2}}\right)=\sum_{(i, j) \in T_{k_{1} \wedge k_{2}, \ell_{1} \wedge \ell_{2}}}\left(\begin{array}{c}
k_{1}+\ell_{1}-i-j \\
k_{1}-i
\end{array}\right)\left(\begin{array}{c}
k_{2}+\ell_{2}-i-j \\
k_{2}-i
\end{array}\right) \alpha^{k_{1}+k_{2}-2 i} \beta^{\ell_{1}+\ell_{2}-2 j},
$$

where $k \wedge \ell:=\min \{k, \ell\}$ and an empty sum is defined to be equal to 0 . Observe, if $0<\alpha<1$ and $\beta=1-\alpha$ then by representation (1.13) covariance (2.1) can be expressed in the form

$$
\operatorname{Cov}\left(X_{k_{1}, \ell_{1}}, X_{k_{2}, \ell_{2}}\right)=\sum_{m=1}^{k_{1} \wedge k_{2}+\ell_{1} \wedge \ell_{2}} \mathrm{P}\left(S_{k_{1}+\ell_{1}-m, k_{2}+\ell_{2}-m}^{(\alpha, 1-\alpha)}=k_{1}+\ell_{2}-m\right)
$$

where for $\nu, \mu \in(0,1)$ real numbers $S_{k, \ell}^{(\mu, \nu)}:=\xi_{k}^{(\mu)}+\eta_{\ell}^{(\nu)}$, and $\xi_{k}^{(\mu)}$ and $\eta_{\ell}^{(\nu)}$ are independent binomial random variables with parameters $(k, \mu)$ and $(\ell, \nu)$, respectively. Now, Lemmas 2.4 and 2.6 of Baran and Pap (2011) directly imply that there exists a constant $D_{\mu, \nu}>0$ such that for all $k, \ell \geq 0, k+\ell \geq 1,0 \leq i \leq k+\ell$ and $0 \leq j \leq k+\ell-1$ we have

$$
\mathrm{P}\left(S_{k, \ell}^{(\mu, \nu)}=i\right) \leq \frac{D_{\mu, \nu}}{\sqrt{k+\ell}} \quad \text { and } \quad\left|\mathrm{P}\left(S_{k, \ell}^{(\mu, \nu)}=j+1\right)-\mathrm{P}\left(S_{k, \ell}^{(\mu, \nu)}=j\right)\right| \leq \frac{D_{\mu, \nu}}{k+\ell} .
$$

Hence, one can determine the magnitudes of the covariances and prove the following lemma.

Lemma 2.1 (Baran et al., 2007, Lemma 2.1) If $|\alpha|+|\beta|=1$ and $0<|\alpha|<1$ then

$$
\left|\operatorname{Cov}\left(X_{k_{1}, \ell_{1}}, X_{k_{2}, \ell_{2}}\right)\right| \leq C_{\alpha} \sqrt{k_{1}+\ell_{1}+k_{2}+\ell_{2}}
$$

with some constant $C_{\alpha}>0$.

Now, for $n \in \mathbb{N}$, let us introduce piecewise constant random fields

$$
Z_{1,0}^{(n)}(s, t):=n^{-1 / 4} X_{[n s]+1,[n t]} \quad \text { and } \quad Z_{0,1}^{(n)}(s, t):=n^{-1 / 4} X_{[n s],[n t]+1}, \quad s, t \in \mathbb{R}, s+t \geq 0 .
$$

Concerning the asymptotic behaviour of their covariances one can verify the following result. 
Proposition 2.2 (Baran et al., 2007, Proposition 2.2) Let $s_{1}, t_{1}, s_{2}, t_{2} \in \mathbb{R}$ with $s_{1}+t_{1}>$ $0, s_{2}+t_{2}>0$. If $0<\alpha<1$ and $\beta=1-\alpha$ then

$$
\left[\begin{array}{ll}
\operatorname{Cov}\left(Z_{1,0}^{(n)}\left(s_{1}, t_{1}\right), Z_{1,0}^{(n)}\left(s_{2}, t_{2}\right)\right) & \operatorname{Cov}\left(Z_{1,0}^{(n)}\left(s_{1}, t_{1}\right), Z_{0,1}^{(n)}\left(s_{2}, t_{2}\right)\right) \\
\operatorname{Cov}\left(Z_{1,0}^{(n)}\left(s_{2}, t_{2}\right), Z_{0,1}^{(n)}\left(s_{1}, t_{1}\right)\right) & \operatorname{Cov}\left(Z_{0,1}^{(n)}\left(s_{1}, t_{1}\right), Z_{0,1}^{(n)}\left(s_{2}, t_{2}\right)\right)
\end{array}\right] \rightarrow z_{\alpha}\left(s_{1}, t_{1}, s_{2}, t_{2}\right)\left[\begin{array}{ll}
1 & 0 \\
0 & 1
\end{array}\right]
$$

as $n \rightarrow \infty$, where

$$
z_{\alpha}\left(s_{1}, t_{1}, s_{2}, t_{2}\right)= \begin{cases}\frac{\sqrt{s_{1}+s_{2}+t_{1}+t_{2}}-\sqrt{\left|s_{1}-s_{2}\right|+\left|t_{1}-t_{2}\right|}}{\sqrt{2 \pi \alpha(1-\alpha)}} & \text { if }(1-\alpha)\left(s_{1}-s_{2}\right)=\alpha\left(t_{1}-t_{2}\right), \\ 0, & \text { otherwise. }\end{cases}
$$

Moreover, if $(1-\alpha)\left(s_{1}-s_{2}\right) \neq \alpha\left(t_{1}-t_{2}\right)$ then the convergence to 0 has an exponential rate.

Further, one can also estimate the difference of two neighbouring covariances.

Proposition 2.3 (Baran et al., 200\%, Proposition 2.5) If $0<\alpha<1$ and $\beta=1-\alpha$ then there exists a constant $K_{\alpha}>0$ such that

$$
\left|\operatorname{Cov}\left(Z_{i, j}^{(n)}\left(s_{1}, t_{1}\right), Z_{j, i}^{(n)}\left(s_{2}, t_{2}\right)\right)-\operatorname{Cov}\left(Z_{i, j}^{(n)}\left(s_{1}, t_{1}\right), Z_{i, j}^{(n)}\left(s_{2}, t_{2}\right)\right)\right| \leq K_{\alpha} n^{-1 / 2}
$$

for all $n \in \mathbb{N}, s_{1}, t_{1}, s_{2}, t_{2} \in \mathbb{R}$, with $s_{1}+t_{1}>0, s_{2}+t_{2}>0$ and $(i, j) \in\{(0,1),(1,0)\}$.

Finally, in order to estimate covariances we make use of he following lemma which is a generalization of Baran et al. (2004, Lemma 11).

Lemma 2.4 (Baran et al., 2007, Lemma 2.8) Let $\xi_{1}, \ldots, \xi_{N}$ be independent random variables with $\mathrm{E}\left(\xi_{i}\right)=0, \mathrm{E}\left(\xi_{i}^{2}\right)=1$ for all $i=1, \ldots, N$, and $M_{4}:=\max _{1 \leq i \leq N} \mathrm{E}\left(\xi_{i}^{4}\right)<\infty$. Let $a_{1}, \ldots, a_{n_{1}}, b_{1}, \ldots, b_{n_{2}}, c_{1}, \ldots, c_{n_{3}}, d_{1}, \ldots, d_{n_{4}} \in \mathbb{R}, n_{1}, n_{2}, n_{3}, n_{4} \leq N$ and

$$
X:=\sum_{i=1}^{n_{1}} a_{i} \xi_{i}, \quad Y:=\sum_{j=1}^{n_{2}} b_{j} \xi_{j}, \quad Z:=\sum_{i=1}^{n_{3}} c_{i} \xi_{i}, \quad W:=\sum_{j=1}^{n_{4}} d_{j} \xi_{j} .
$$

Then

$$
\operatorname{Cov}(X Y, Z W)=\sum_{i=1}^{n_{1} \wedge n_{2} \wedge n_{3} \wedge n_{4}}\left(\mathrm{E}\left(\xi_{i}^{4}\right)-3\right) a_{i} b_{i} c_{i} d_{i}+\operatorname{Cov}(X, Z) \operatorname{Cov}(Y, W)+\operatorname{Cov}(X, W) \operatorname{Cov}(Y, Z) .
$$

Moreover, if $a_{i}, b_{i}, c_{i}, d_{i} \geq 0$ then

$$
0 \leq \operatorname{Cov}(X Y, Z W) \leq M_{4} \operatorname{Cov}(X, Z) \operatorname{Cov}(Y, W)+M_{4} \operatorname{Cov}(X, W) \operatorname{Cov}(Y, Z),
$$

and

$$
0 \leq \mathrm{E}(X Y Z W) \leq M_{4}(\mathrm{E}(X Z) \mathrm{E}(Y W)+\mathrm{E}(X W) \mathrm{E}(Y Z)+\mathrm{E}(X Y) \mathrm{E}(Z W))
$$




\section{Proof of Proposition 1.2}

Let $\alpha, \beta \in(0,1)$ with $\alpha+\beta=1$ and

$S_{n, 1}:=\sum_{(k, \ell) \in T_{n}}\left(X_{k-1, \ell}-X_{k, \ell-1}\right)^{2}, \quad S_{n, 2}:=\sum_{(k, \ell) \in T_{n}}\left(X_{k-1, \ell}-X_{k, \ell-1}\right) X_{k, \ell-1}, \quad S_{n, 3}:=\sum_{(k, \ell) \in T_{n}} X_{k, \ell-1}^{2}$.

Thus,

$$
\widetilde{A}_{T_{n}}=\left[\begin{array}{cc}
n^{-2} S_{n, 1} & n^{-9 / 4} S_{n, 2} \\
n^{-9 / 4} S_{n, 2} & n^{-5 / 2} S_{n, 3}
\end{array}\right]
$$

and (1.9) follows from

$$
n^{-2} S_{n, 1} \stackrel{\mathrm{L}_{2}}{\longrightarrow} \frac{1}{\varphi_{\alpha}}=\frac{2}{\alpha(1-\alpha)}, \quad n^{-9 / 4} S_{n, 2} \stackrel{\mathrm{L}_{2}}{\longrightarrow} 0, \quad n^{-2} S_{n, 3} \stackrel{\mathrm{L}_{2}}{\longrightarrow} \frac{1}{\psi_{\alpha}}=\frac{2^{9 / 2}}{15 \sqrt{\pi \alpha(1-\alpha)}}
$$

The last two statements of (3.1) have already been proved, see Baran et al. (2007, Proposition 1.2 and Section 6, pp. 40-41). In order to verify the remaining statement one has to show

$$
\lim _{n \rightarrow \infty} n^{-2} \mathrm{E}\left(S_{n, 1}\right)=\frac{1}{\varphi_{\alpha}} \quad \text { and } \quad \lim _{n \rightarrow \infty} n^{-4} \operatorname{Var}\left(S_{n, 1}\right)=0
$$

It is easy to see that

$$
n^{-2} \mathrm{E}\left(S_{n, 1}\right)=\iint_{T} \sqrt{n}\left(\operatorname{Var}\left(Z_{0,1}^{(n)}(s, t)\right)+\operatorname{Var}\left(Z_{1,0}^{(n)}(s, t)\right)-2 \operatorname{Cov}\left(Z_{0,1}^{(n)}(s, t), Z_{1,0}^{(n)}(s, t)\right)\right) \mathrm{d} s \mathrm{~d} t
$$

where $T:=\left\{(s, t) \in \mathbb{R}^{2}: s+t \geq 0, s \leq 1, t \leq 1\right\}$, and using (2.1) one can prove

$$
\lim _{n \rightarrow \infty} \sqrt{n}\left(\operatorname{Var}\left(Z_{i, j}^{(n)}(s, t)\right)-\operatorname{Cov}\left(Z_{i, j}^{(n)}(s, t), Z_{j, i}^{(n)}(s, t)\right)\right)=\frac{1}{2 \alpha(1-\alpha)}
$$

where $(i, j) \in\{(0,1),(1,0)\}$. The details can be found in Baran et al. (2007, Section 5, pp. 36-37). Hence, Proposition 2.3 and the dominated convergence theorem imply the first statement of (3.2).

Now, by Lemma 2.4

$$
\begin{aligned}
\operatorname{Var}\left(S_{n, 1}\right) & =\sum_{\left(k_{1}, \ell_{1}\right) \in T_{n}} \sum_{\left(k_{2}, \ell_{2}\right) \in T_{n}} \operatorname{Cov}\left(\left(X_{k_{1}-1, \ell_{1}}-X_{k_{1}, \ell_{1}-1}\right)^{2},\left(X_{k_{2}-1, \ell_{2}}-X_{k_{2}, \ell_{2}-1}\right)^{2}\right) \\
& \leq \sum_{\left(k_{1}, \ell_{1}\right) \in T_{n}} \sum_{\left(k_{2}, \ell_{2}\right) \in T_{n}}\left(2 M_{4} L_{k_{1}, \ell_{1}, k_{2}, \ell_{2}}^{(1)}+\left(M_{4}-3\right)^{+} L_{k_{1}, \ell_{1}, k_{2}, \ell_{2}}^{(2)}\right)+\mathcal{O}\left(n^{3}\right),
\end{aligned}
$$


where

$$
\begin{aligned}
L_{k_{1}, \ell_{1}, k_{2}, \ell_{2}}^{(1)} & :=\operatorname{Cov}\left(X_{k_{1}-1, \ell_{1}}-X_{k_{1}, \ell_{1}-1}, X_{k_{2}-1, \ell_{2}}-X_{k_{2}, \ell_{2}-1}\right)^{2}, \\
L_{k_{1}, \ell_{1}, k_{2}, \ell_{2}}^{(2)}:=\sum_{(i, j) \in T_{k_{1} \wedge k_{2}-1, \ell_{1} \wedge \ell_{2}-1}}\left(\mathrm{P}\left(S_{k_{1}+\ell_{1}-1-i-j}^{(\alpha)}=k_{1}-i\right)-\mathrm{P}\left(S_{k_{1}+\ell_{1}-1-i-j}^{(\alpha)}=k_{1}-1-i\right)\right)^{2} & (3.3) \\
\quad \times\left(\mathrm{P}\left(S_{k_{2}+\ell_{2}-1-i-j}^{(\alpha)}=k_{2}-i\right)-\mathrm{P}\left(S_{k_{2}+\ell_{2}-1-i-j}^{(\alpha)}=k_{2}-1-i\right)\right)^{2} & \left(\mathrm{P}\left(S_{k_{1}+\ell_{1}-1-i-j}^{(\alpha)}=k_{1}-i\right)^{2}+\mathrm{P}\left(S_{k_{1}+\ell_{1}-1-i-j}^{(\alpha)}=k_{1}-1-i\right)^{2}\right) \\
\leq & \sum_{(i, j) \in T_{k_{1} \wedge k_{2}-1, \ell_{1} \wedge \ell_{2}-1}} \\
\times & \left(\mathrm{P}\left(S_{k_{2}+\ell_{2}-1-i-j}^{(1-\alpha)}=\ell_{2}-1-j\right)^{2}+\mathrm{P}\left(S_{k_{2}+\ell_{2}-1-i-j}^{(1-\alpha)}=\ell_{2}-j\right)^{2}\right) .
\end{aligned}
$$

Obviously,

$$
\begin{aligned}
& n^{-4} \sum_{\left(k_{1}, \ell_{1}\right) \in T_{n}} \sum_{\left(k_{2}, \ell_{2}\right) \in T_{n}} L_{k_{1}, \ell_{1}, k_{2}, \ell_{2}}^{(1)} \\
& \quad=\iint_{T} \iint_{T}\left(\sqrt{n} \operatorname{Cov}\left(Z_{0,1}^{(n)}\left(s_{1}, t_{1}\right)-Z_{1,0}^{(n)}\left(s_{1}, t_{1}\right), Z_{0,1}^{(n)}\left(s_{2}, t_{2}\right)-Z_{1,0}^{(n)}\left(s_{2}, t_{2}\right)\right)\right)^{2} \mathrm{~d} s_{1} \mathrm{~d} t_{1} \mathrm{~d} s_{2} \mathrm{~d} t_{2},
\end{aligned}
$$

where due to Propositions 2.2, 2.3 and dominated convergence theorem the right hand side converges to 0 as $n \rightarrow \infty$.

Further, the second inequality of (2.2) implies

$$
\begin{aligned}
L_{k_{1}, \ell_{1}, k_{2}, \ell_{2}}^{(2)} & \leq \sum_{(i, j) \in T_{k_{1} \wedge k_{2}-1, \ell_{1} \wedge \ell_{2}-1}} \frac{D_{\alpha, \alpha}^{4}}{\left(k_{1}+\ell_{1}-1-i-j\right)^{2}\left(k_{2}+\ell_{2}-1-i-j\right)^{2}} \\
& \leq \sum_{m=1}^{k_{1} \wedge k_{2}+\ell_{1} \wedge \ell_{2}-2} \frac{D_{\alpha, \alpha}^{4}\left(k_{1} \wedge k_{2}+\ell_{1} \wedge \ell_{2}-1-m\right)}{\left(k_{1}+\ell_{1}-1-m\right)^{2}\left(k_{2}+\ell_{2}-1-m\right)^{2}} \\
& \leq \sum_{m=1}^{k_{1} \wedge k_{2}+\ell_{1} \wedge \ell_{2}-2} \frac{D_{\alpha, \alpha}^{4}}{\left(k_{1} \wedge k_{2}+\ell_{1} \wedge \ell_{2}-1-m\right)^{3}}<\sum_{m=1}^{k_{1} \wedge k_{2}+\ell_{1} \wedge \ell_{2}-2} \frac{D_{\alpha, \alpha}^{4}}{m^{2}}<\frac{\pi^{2} D_{\alpha, \alpha}^{4}}{6}<\infty,
\end{aligned}
$$

So

$$
n^{-4} \sum_{\left(k_{1}, \ell_{1}\right) \in T_{n}} \sum_{\left(k_{2}, \ell_{2}\right) \in T_{n}} L_{k_{1}, \ell_{1}, k_{2}, \ell_{2}}^{(2)}=\iiint_{T} \iint_{T} L_{\left[n s_{1}\right],\left[n t_{1}\right],\left[n s_{2}\right],\left[n t_{2}\right]}^{(2)} \mathrm{d} s_{1} \mathrm{~d} t_{1} \mathrm{~d} s_{2} \mathrm{~d} t_{2} \leq \frac{2 \pi^{2} D_{\alpha, \alpha}^{4}}{3} .
$$

Finally, e.g.

$$
\begin{gathered}
\sum_{(i, j) \in T_{\left[n s_{1}\right] \wedge\left[n s_{2}\right]-1,\left[n t_{1}\right] \wedge\left[n t_{2}\right]-1}} \mathrm{P}\left(S_{\left[n s_{1}\right]+\left[n t_{1}\right]-1-i-j}^{(\alpha)}=\left[n s_{1}\right]-i\right)^{2} \mathrm{P}\left(S_{\left[n s_{2}\right]+\left[n t_{2}\right]-1-i-j}^{(1-\alpha)}=\left[n t_{2}\right]-1-j\right)^{2} \\
\leq \sqrt{n} \operatorname{Cov}\left(Z_{1,0}^{(n)}\left(s_{1}, t_{1}\right)-Z_{1,0}^{(n)}\left(s_{2}, t_{2}\right)\right)
\end{gathered}
$$

which by Proposition 2.2 converges to 0 as $n \rightarrow \infty$ if $(1-\alpha)\left(s_{1}-s_{2}\right) \neq \alpha\left(t_{1}-t_{2}\right)$. Similar results can be derived for the remaining three terms of the right hand side of (3.3), 
so by the dominated convergence theorem

$$
\lim _{n \rightarrow \infty} n^{-4} \sum_{\left(k_{1}, \ell_{1}\right) \in T_{n}} \sum_{\left(k_{2}, \ell_{2}\right) \in T_{n}} L_{k_{1}, \ell_{1}, k_{2}, \ell_{2}}^{(2)}=0,
$$

which completes the proof.

\section{Proof of Proposition 1.3}

Again, let $\alpha, \beta \in(0,1)$ with $\alpha+\beta=1$ and denote by $d_{n}^{(i)}, i=1,2$, the components of $d_{T_{n}}$. First we show that $\left(d_{T_{n}}\right)_{n \geq 1}$ is a square integrable two dimensional martingale with respect to filtration $\left(\mathcal{F}_{n}\right)_{n \geq 1}$, where $\mathcal{F}_{n}$ denotes the $\sigma$-algebra generated by random variables $\left\{\varepsilon_{k, \ell}:(k, \ell) \in T_{n}\right\}$.

In order to do this we give a useful decomposition of $d_{T_{n}}-d_{T_{n-1}}$, where $d_{T_{0}}:=(0,0)^{\top}$. By representation (1.12),

$$
\begin{aligned}
d_{n}^{(1)}-d_{n-1}^{(1)}= & \sum_{(k, \ell) \in T_{n} \backslash T_{n-1}} \varepsilon_{k, \ell}\left(\sum_{(i, j) \in T_{k-1, \ell}} \mathrm{P}\left(S_{k+\ell-1-i-j}^{(\alpha)}=k-1-i\right) \varepsilon_{i, j}\right. \\
& \left.-\sum_{(i, j) \in T_{k, \ell-1}} \mathrm{P}\left(S_{k+\ell-1-i-j}^{(\alpha)}=k-i\right) \varepsilon_{i, j}\right), \\
d_{n}^{(2)}-d_{n-1}^{(2)}= & \sum_{(k, \ell) \in T_{n} \backslash T_{n-1}} \varepsilon_{k, \ell} \sum_{(i, j) \in T_{k, \ell-1}} \mathrm{P}\left(S_{k+\ell-1-i-j}^{(\alpha)}=k-i\right) \varepsilon_{i, j} .
\end{aligned}
$$

Collecting first the terms containing only $\varepsilon_{i, j}$ with $(i, j) \in T_{n} \backslash T_{n-1}$, and then the rest, we obtain decomposition

$$
d_{T_{n}}-d_{T_{n-1}}=d_{n, 1}+\sum_{(k, \ell) \in T_{n} \backslash T_{n-1}} \varepsilon_{k, \ell} d_{n, 2, k, \ell},
$$

where $d_{n, 1}=\left(\delta_{n, 1}^{(1)}-\delta_{n, 1}^{(2)}, \delta_{n, 1}^{(2)}\right)^{\top}$ and $d_{n, 2, k, \ell}=\left(\delta_{n, 2, k-1, \ell}-\delta_{n, 2, k, \ell-1}, \delta_{n, 2, k, \ell-1}\right)^{\top}$ with

$$
\begin{aligned}
\delta_{n, 1}^{(1)} & :=\sum_{(k, \ell) \in T_{n} \backslash T_{n-1}, \ell} \varepsilon_{k, j) \in T_{k-1, \ell} \backslash T_{n-1}} \mathrm{P}\left(S_{k+\ell-1-i-j}^{(\alpha)}=k-1-i\right) \varepsilon_{i, j}=\sum_{k=-n+2}^{m} \sum_{i=-n+1}^{k-1} \alpha^{k-1-i} \varepsilon_{k, n} \varepsilon_{i, n}, \\
\delta_{n, 1}^{(2)} & :=\sum_{(k, \ell) \in T_{n} \backslash T_{n-1}, \ell} \varepsilon_{k, \ell} \sum_{(i, j) \in T_{k, \ell-1} \backslash T_{n-1}} \mathrm{P}\left(S_{k+\ell-1-i-j}^{(\alpha)}=k-i\right) \varepsilon_{i, j}=\sum_{\ell=-n+2}^{n} \sum_{j=-n+1}^{\ell-1} \beta^{\ell-1-j} \varepsilon_{n, \ell} \varepsilon_{n, j}, \\
\delta_{n, 2, k, \ell} & :=\sum_{(i, j) \in T_{k, \ell} \cap T_{n-1}} \mathrm{P}\left(S_{k+\ell-i-j}^{(\alpha)}=k-i\right) \varepsilon_{i, j} .
\end{aligned}
$$

The components of $d_{n, 1}$ are quadratic forms of the variables $\left\{\varepsilon_{i, j}:(i, j) \in T_{n} \backslash T_{n-1}\right\}$, hence $d_{n, 1}$ is independent of $\mathcal{F}_{n-1}$. Besides this the terms $\delta_{n, 2, k, \ell}$ are linear combinations 
of the variables $\left\{\varepsilon_{i, j}:(i, j) \in T_{n-1}\right\}$, thus vectors $d_{n, 2, k, \ell}$ are measurable with respect to $\mathcal{F}_{n-1}$. Consequently,

$$
\mathrm{E}\left(d_{T_{n}}-d_{T_{n-1}} \mid \mathcal{F}_{n-1}\right)=\mathrm{E}\left(d_{n, 1}\right)+\sum_{(k, \ell) \in T_{n} \backslash T_{n-1}} d_{n, 2, k, \ell} \mathrm{E}\left(\varepsilon_{k, \ell} \mid \mathcal{F}_{n-1}\right)=0
$$

Hence $\left(d_{T_{n}}\right)_{n \geq 1}$ is a square integrable martingale with respect to the filtration $\left(\mathcal{F}_{n}\right)_{n \geq 1}$ and obviously the same is valid for $\left(\widetilde{d}_{T_{n}}\right)_{n \geq 1}$.

By the Martingale Central Limit Theorem (Jacod and Shiryaev, 1987), in order to prove the statement of Proposition 1.3, it suffices to show that the conditional variances of the martingale differences converge in probability and to verify the conditional Lindeberg condition. To be precise, the statement is a consequence of the following two propositions, where $\mathbb{1}_{H}$ denotes the indicator function of a set $H$.

Proposition 4.1

$$
\sum_{m=1}^{n} \mathrm{E}\left(\left(\widetilde{d}_{T_{m}}-\widetilde{d}_{T_{m-1}}\right)\left(\widetilde{d}_{T_{m}}-\widetilde{d}_{T_{m-1}}\right)^{\top} \mid \mathcal{F}_{m-1}\right) \stackrel{\mathrm{P}}{\longrightarrow} \widetilde{A} \quad \text { as } n \rightarrow \infty .
$$

Proposition 4.2 For all $\delta>0$,

$$
\sum_{m=1}^{n} \mathrm{E}\left(\left\|\widetilde{d}_{T_{m}}-\widetilde{d}_{T_{m-1}}\right\|^{2} \mathbb{1}_{\left\{\left\|\widetilde{d}_{T_{m}}-\widetilde{d}_{T_{m-1}}\right\| \geq \delta\right\}} \mid \mathcal{F}_{m-1}\right) \stackrel{\mathrm{P}}{\longrightarrow} 0 \quad \text { as } n \rightarrow \infty .
$$

Proof of Proposition 4.1. Considering separately the entries of $\left(\widetilde{d}_{T_{m}}-\widetilde{d}_{T_{m-1}}\right)\left(\widetilde{d}_{T_{m}}-\right.$ $\left.\widetilde{d}_{T_{m-1}}\right)^{\top}$ one can see that the statement of the proposition is a consequence of

$$
\begin{gathered}
n^{-2} \sum_{m=1}^{n} \mathrm{E}\left(\left(d_{m}^{(1)}-d_{m-1}^{(1)}\right)^{2} \mid \mathcal{F}_{m-1}\right) \stackrel{\mathrm{L}_{2}}{\longrightarrow} \frac{1}{\varphi_{\alpha}}, \\
n^{-5 / 2} \sum_{m=1}^{n} \mathrm{E}\left(\left(d_{m}^{(2)}-d_{m-1}^{(2)}\right)^{2} \mid \mathcal{F}_{m-1}\right) \stackrel{\mathrm{L}_{2}}{\longrightarrow} \frac{1}{\psi_{\alpha}}, \\
n^{-9 / 4} \sum_{m=1}^{n} \mathrm{E}\left(\left(d_{m}^{(1)}-d_{m-1}^{(1)}\right)\left(d_{m}^{(2)}-d_{m-1}^{(2)}\right) \mid \mathcal{F}_{m-1}\right) \stackrel{\mathrm{L}_{2}}{\longrightarrow} 0
\end{gathered}
$$

as $n \rightarrow \infty$. Limits (4.2) and (4.3) have already been proved, see Baran et al. (2007, Section 6, pp. 40-41 and Proposition 4.1). A more detailed proof can be found in Baran et al. (2005, Propositions 6.1 and 4.1).

Now, let $U_{m}:=\mathrm{E}\left(\left(d_{m}^{(1)}-d_{m-1}^{(1)}\right)\left(d_{m}^{(2)}-d_{m-1}^{(2)}\right) \mid \mathcal{F}_{m-1}\right)$ and we have

$$
d_{m}^{(1)}-d_{m-1}^{(1)}=\sum_{(k, \ell) \in T_{m} \backslash T_{m-1}}\left(X_{k-1, \ell}-X_{k, \ell-1}\right) \varepsilon_{k, \ell}, \quad d_{m}^{(2)}-d_{m-1}^{(2)}=\sum_{(k, \ell) \in T_{m} \backslash T_{m-1}} X_{k, \ell-1} \varepsilon_{k, \ell} .
$$


Representation (1.12) and independence of the error terms $\varepsilon_{i, j}$ imply

$$
\begin{aligned}
\mathrm{E}\left(\left(d_{m}^{(1)}-d_{m-1}^{(1)}\right)\right. & \left.\left(d_{m}^{(2)}-d_{m-1}^{(2)}\right)\right)=\sum_{(k, \ell) \in T_{m} \backslash T_{m-1}} \mathrm{E}\left(\left(X_{k-1, \ell}-X_{k, \ell-1}\right) X_{k, \ell-1}\right) \mathrm{E}\left(\varepsilon_{k, \ell}^{2}\right) \\
= & \sum_{(k, \ell) \in T_{m} \backslash T_{m-1}} \mathrm{E}\left(\left(X_{k-1, \ell}-X_{k, \ell-1}\right) X_{k, \ell-1}\right)=\mathrm{E}\left(S_{m, 2}\right)-\mathrm{E}\left(S_{m-1,2}\right),
\end{aligned}
$$

so using the second statement of (3.1) we obtain

$$
n^{-9 / 4} \sum_{m=1}^{n} \mathrm{E}\left(U_{m}\right)=n^{-9 / 4} \mathrm{E}\left(S_{n, 2}\right) \rightarrow 0 \quad \text { as } n \rightarrow \infty .
$$

Further, decomposition (4.1), independence of $\delta_{m, 1}^{(1)}, \delta_{m, 1}^{(2)}$ and $\left\{\varepsilon_{k, \ell},(k, \ell) \in T_{m} \backslash T_{m-1}\right\}$ from $\mathcal{F}_{m-1}$, and measurability of $\delta_{m, 2, k, \ell}$ with respect to $\mathcal{F}_{m-1}$ imply

$$
U_{m}=\mathrm{E}\left(\left(\delta_{m, 1}^{(1)}-\delta_{m, 1}^{(2)}\right) \delta_{m, 1}^{(2)}\right)+\sum_{(k, \ell) \in T_{m} \backslash T_{m-1}}\left(\delta_{m, 2, k-1, \ell}-\delta_{m, 2, k, \ell-1}\right) \delta_{m, 2, k, \ell-1}
$$

In this way, to complete the proof of (4.4) one has to show

$$
n^{-9 / 2} \operatorname{Var}\left(\sum_{m=1}^{n} U_{m}\right)=n^{-9 / 2} \operatorname{Var}\left(\sum_{m=1}^{n} \sum_{(k, \ell) \in T_{m} \backslash T_{m-1}}\left(\delta_{m, 2, k-1, \ell}-\delta_{m, 2, k, \ell-1}\right) \delta_{m, 2, k, \ell-1}\right) \rightarrow 0
$$

as $n \rightarrow \infty$.

Now, consider

$$
\begin{aligned}
\operatorname{Var} & \left(\sum_{m=1}^{n} \sum_{(k, \ell) \in T_{m} \backslash T_{m-1}}\left(\delta_{m, 2, k-1, \ell}-\delta_{m, 2, k, \ell-1}\right) \delta_{m, 2, k, \ell-1}\right) \\
= & \sum_{m_{1}=1}^{n} \sum_{\left(k_{1}, \ell_{1}\right) \in T_{m_{1}} \backslash T_{m_{1}-1}} \sum_{m_{2}=1}^{n} \sum_{\left(k_{2}, \ell_{2}\right) \in T_{m_{2}} \backslash T_{m_{2}-1}} G_{m_{1}, m_{2}, k_{1}, \ell_{1}, k_{2}, \ell_{2}} \\
= & \sum_{m_{1}=1}^{n} \sum_{m_{2}=1}^{n}\left(\sum_{k_{1}=-m_{1}+1}^{m_{1}} \sum_{k_{2}=-m_{2}+1}^{m_{2}} G_{m_{1}, m_{2}, k_{1}, m_{1}, k_{2}, m_{2}}+\sum_{k_{1}=-m_{1}+1}^{m_{1}} \sum_{\ell_{2}=-m_{2}+1}^{m_{2}-1} G_{m_{1}, m_{2}, k_{1}, m_{1}, m_{2}, \ell_{2}}\right. \\
& \left.\quad+\sum_{\ell_{1}=-m_{1}+1}^{m_{1}-1} \sum_{k_{2}=-m_{2}+1}^{m_{2}} G_{m_{1}, m_{2}, m_{1}, \ell_{1}, k_{2}, m_{2}}+\sum_{\ell_{1}=-m_{1}+1}^{m_{1}-1} \sum_{\ell_{2}=-m_{2}+1}^{m_{2}-1} G_{m_{1}, m_{2}, m_{1}, \ell_{1}, m_{2}, \ell_{2}}\right),
\end{aligned}
$$

where

$$
\begin{aligned}
& G_{m_{1}, m_{2}, k_{1}, \ell_{1}, k_{2}, \ell_{2}} \\
& \quad:=\operatorname{Cov}\left(\left(\delta_{m_{1}, 2, k_{1}-1, \ell_{1}}-\delta_{m_{1}, 2, k_{1}, \ell_{1}-1}\right) \delta_{m_{1}, 2, k_{1}, \ell_{1}-1},\left(\delta_{m_{2}, 2, k_{2}-1, \ell_{2}}-\delta_{m_{2}, 2, k_{2}, \ell_{2}-1}\right) \delta_{m_{2}, 2, k_{2}, \ell_{2}-1}\right) .
\end{aligned}
$$


By representation (1.12) of $X_{k, \ell}$ and definition of $\delta_{m, 2, k, \ell}$ we have

$$
\begin{array}{lll}
\delta_{m, 2, k-1, m}=X_{k-1, m}-\sum_{i=-m+2}^{k-1} \alpha^{k-1-i} \varepsilon_{i, m}, & -m+2 \leq k \leq m, \\
\delta_{m, 2, k, m-1}=X_{k, m-1}, & -m+1 \leq k \leq m, \\
\delta_{m, 2, m, \ell-1}=X_{m, \ell-1}-\sum_{j=-m+2}^{\ell-1}(1-\alpha)^{\ell-1-j} \varepsilon_{m, j}, & -m+2 \leq \ell \leq m-1, \\
\delta_{m, 2, m-1, \ell}=X_{m-1, \ell}, & -m+1 \leq \ell \leq m-1 .
\end{array}
$$

Hence, e.g.

$$
\begin{aligned}
& \sum_{k_{1}=-m_{1}+1}^{m_{1}} \sum_{k_{2}=-m_{2}+1}^{m_{2}} G_{m_{1}, m_{2}, k_{1}, m_{1}, k_{2}, m_{2}} \\
& =\sum_{k_{1}=-m_{1}+1}^{m_{1}} \sum_{k_{2}=-m_{2}+1}^{m_{2}} \operatorname{Cov}\left(\left(X_{k_{1}-1, m_{1}}-X_{k_{1}, m_{1}-1}-\sum_{i_{1}=-m_{1}+2}^{k_{1}-1} \alpha^{k_{1}-1-i_{1}} \varepsilon_{i_{1}, m_{1}}\right) X_{k_{1}, m_{1}-1},\right. \\
& \left.\quad\left(X_{k_{2}-1, m_{2}}-X_{k_{2}, m_{2}-1}-\sum_{i_{2}=-m_{2}+2}^{k_{2}-1} \alpha^{k_{2}-1-i_{2}} \varepsilon_{i_{2}, m_{2}}\right) X_{k_{2}, m_{2}-1}\right) \\
& =\sum_{k_{1}=-m_{1}+1}^{m_{1}} \sum_{k_{2}=-m_{2}+1}^{m_{2}} G_{k_{1}, m_{1}, k_{2}, m_{2}}^{(1)}-G_{k_{1}, m_{1}, k_{2}, m_{2}}^{(2)}-G_{k_{2}, m_{2}, k_{1}, m_{1}}^{(2)}+G_{k_{1}, m_{1}, k_{2}, m_{2}}^{(3)},
\end{aligned}
$$

where

$$
\begin{aligned}
G_{k_{1}, m_{1}, k_{2}, m_{2}}^{(1)} & :=\operatorname{Cov}\left(\left(X_{k_{1}-1, m_{1}}-X_{k_{1}, m_{1}-1}\right) X_{k_{1}, m_{1}-1},\left(X_{k_{2}-1, m_{2}}-X_{k_{2}, m_{2}-1}\right) X_{k_{2}, m_{2}-1}\right), \\
G_{k_{1}, m_{1}, k_{2}, m_{2}}^{(2)} & :=\operatorname{Cov}\left(\left(X_{k_{1}-1, m_{1}}-X_{k_{1}, m_{1}-1}\right) X_{k_{1}, m_{1}-1}, X_{k_{2}, m_{2}-1} \sum_{i=-m_{2}+2}^{k_{2}-1} \alpha^{k_{2}-1-i} \varepsilon_{i, m_{2}}\right), \\
G_{k_{1}, m_{1}, k_{2}, m_{2}}^{(3)} & :=\operatorname{Cov}\left(X_{k_{1}, m_{1}-1} \sum_{i_{1}=-m_{1}+2}^{k_{1}-1} \alpha^{k_{1}-1-i_{1}} \varepsilon_{i_{1}, m_{1}}, X_{k_{2}, m_{2}-1} \sum_{i_{2}=-m_{2}+2}^{k_{2}-1} \alpha^{k_{2}-1-i_{2}} \varepsilon_{i_{2}, m_{2}}\right) .
\end{aligned}
$$

Thus, Lemma 2.4, representation (1.12) and independence of the error terms $\varepsilon_{i, j}$ imply

$$
\begin{aligned}
G_{k_{1}, m_{1}, k_{2}, m_{2}}^{(2)}= & \operatorname{Cov}\left(X_{k_{1}-1, m_{1}}-X_{k_{1}, m_{1}-1}, X_{k_{2}, m_{2}-1}\right) \operatorname{Cov}\left(X_{k_{1}, m_{1}-1}, \sum_{i=-m_{2}+2}^{k_{2}-1} \alpha^{k_{2}-1-i} \varepsilon_{i, m_{2}}\right) \\
& +\operatorname{Cov}\left(X_{k_{1}, m_{1}-1}, X_{k_{2}, m_{2}-1}\right) \operatorname{Cov}\left(X_{k_{1}-1, m_{1}}-X_{k_{1}, m_{1}-1}, \sum_{i=-m_{2}+2}^{k_{2}-1} \alpha^{k_{2}-1-i} \varepsilon_{i, m_{2}}\right), \\
G_{k_{1}, m_{1}, k_{2}, m_{2}}^{(3)}= & \operatorname{Cov}\left(X_{k_{1}, m_{1}-1}, X_{k_{2}, m_{2}-1}\right) \operatorname{Cov}\left(\sum_{i_{1}=-m_{1}+2}^{k_{1}-1} \alpha^{k_{1}-1-i_{1}} \varepsilon_{i_{1}, m_{1}}, \sum_{i_{2}=-m_{2}+2}^{k_{2}-1} \alpha^{k_{2}-1-i_{2}} \varepsilon_{i_{2}, m_{2}}\right) \\
& +\operatorname{Cov}\left(X_{k_{1}, m_{1}-1}, \sum_{i_{2}=-m_{2}+2}^{k_{2}-1} \alpha^{k_{2}-1-i_{2}} \varepsilon_{i_{2}, m_{2}}\right) \operatorname{Cov}\left(X_{k_{2}, m_{2}-1}, \sum_{i_{1}=-m_{1}+2}^{k^{k_{1}-1-i_{1}}} \varepsilon_{i_{1}, m_{1}}\right) .
\end{aligned}
$$


Moreover, using again the independence of the error terms $\varepsilon_{i, j}$ one can easily see that $G_{k_{1}, m_{1}, k_{2}, m_{2}}^{(2)}=0$ if $m_{2}>m_{1}$ and $G_{k_{1}, m_{1}, k_{2}, m_{2}}^{(3)}=0$ if $m_{2} \neq m_{1}$. In this way

$$
\begin{aligned}
& \sum_{m_{1}=1}^{n} \sum_{m_{2}=1}^{n} \sum_{k_{1}=-m_{1}+1}^{m_{1}} \sum_{k_{2}=-m_{2}+1}^{m_{2}} G_{k_{1}, m_{1}, k_{2}, m_{2}}^{(3)} \\
& \quad=\sum_{m=1}^{n} \sum_{k_{1}=-m+1}^{m} \sum_{k_{2}=-m+1}^{m} \operatorname{Cov}\left(X_{k_{1}, m-1}, X_{k_{2}, m-1}\right) \alpha^{\left|k_{1}-k_{2}\right|} \sum_{i=0}^{m+k_{1} \wedge k_{2}-3} \alpha^{2 i} \\
& \quad \leq \frac{C_{\alpha}}{1-\alpha^{2}} \sum_{m=1}^{n} \sum_{k_{1}=0}^{2 m-1} \sum_{k_{2}=0}^{2 m-1}\left(k_{1}+k_{2}\right)^{1 / 2} \leq \frac{3 C_{\alpha}}{1-\alpha^{2}}(n+1)^{7 / 2}
\end{aligned}
$$

where the first inequality is a consequence of Lemma 2.1 and the empty sum is defined to be zero.

Further, let

$$
\begin{aligned}
& B_{k_{1}, m_{1}, k_{2}, m_{2}}^{(1)}:=\operatorname{Cov}\left(X_{k_{1}, m_{1}-1}, \sum_{i=-m_{2}+2}^{k_{2}-1} \alpha^{k_{2}-1-i} \varepsilon_{i, m_{2}}\right), \\
& B_{k_{1}, m_{1}, k_{2}, m_{2}}^{(2)}:=\operatorname{Cov}\left(X_{k_{1}-1, m_{1}}-X_{k_{1}, m_{1}-1}, \sum_{i=-m_{2}+2}^{k_{2}-1} \alpha^{k_{2}-1-i} \varepsilon_{i, m_{2}}\right) .
\end{aligned}
$$

Assuming $m_{2}<m_{1}$, with the help of representation (1.13) we obtain

$$
\begin{aligned}
B_{k_{1}, m_{1}, k_{2}, m_{2}}^{(1)}= & \sum_{i=-m_{2}+2}^{k_{1} \wedge k_{2}-1} \mathrm{P}\left(S_{k_{1}+m_{1}-m_{2}-1-i}^{(\alpha)}=k_{1}-i\right) \alpha^{k_{2}-1-i}+\alpha^{k_{2}-k_{1}-1}(1-\alpha)^{m_{1}-m_{2}-1} \mathbb{1}_{\left\{k_{1} \leq k_{2}-1\right\}}, \\
B_{k_{1}, m_{1}, k_{2}, m_{2}}^{(2)}= & \sum_{i=-m_{2}+2}^{k_{1} \wedge k_{2}-1}\left(\mathrm{P}\left(S_{k_{1}+m_{1}-m_{2}-1-i}^{(\alpha)}=k_{1}-1-i\right)-\mathrm{P}\left(S_{k_{1}+m_{1}-m_{2}-1-i}^{(\alpha)}=k_{1}-i\right)\right) \alpha^{k_{2}-1-i} \\
& -\alpha^{k_{2}-k_{1}-1}(1-\alpha)^{m_{1}-m_{2}-1} \mathbb{1}_{\left\{k_{1} \leq k_{2}-1\right\}}
\end{aligned}
$$

for $k_{1}+m_{1} \geq 3$, otherwise the above quantities are equal to zero. Hence, using (2.2) one can easily show that for $k_{1} \leq k_{2}-1$

$$
\begin{aligned}
\left|B_{k_{1}, m_{1}, k_{2}, m_{2}}^{(1)}\right| & \leq \alpha^{k_{2}-k_{1}-1}(1-\alpha)^{m_{1}-m_{2}-1}+\alpha^{k_{2}-k_{1}} \sum_{i=-m_{2}+2}^{k_{1}-1} \frac{D_{\alpha, \alpha}}{\left(k_{1}+m_{1}-m_{2}-1-i\right)^{1 / 2}} \\
& \leq H_{\alpha} \alpha^{k_{2}-k_{1}}\left(k_{1}+m_{1}\right)^{1 / 2}, \\
\left|B_{k_{1}, m_{1}, k_{2}, m_{2}}^{(2)}\right| & \leq \alpha^{k_{2}-k_{1}-1}(1-\alpha)^{m_{1}-m_{2}-1}+\alpha^{k_{2}-k_{1}} \sum_{i=-m_{2}+2}^{k_{1}-1} \frac{D_{\alpha, \alpha}}{k_{1}+m_{1}-m_{2}-1-i} \\
& \leq H_{\alpha} \alpha^{k_{2}-k_{1}} \log \left(k_{1}+m_{1}\right)
\end{aligned}
$$


with some constant $H_{\alpha}>0$, while for $k_{1}>k_{2}-1$ we have

$$
\begin{aligned}
& \left|B_{k_{1}, m_{1}, k_{2}, m_{2}}^{(1)}\right| \leq \frac{D_{\alpha, \alpha}}{\left(k_{1}-k_{2}+m_{1}-m_{2}\right)^{1 / 2}} \sum_{i=-m_{2}+2}^{k_{2}-1} \alpha^{k_{2}-1-i} \leq \frac{H_{\alpha}}{\left(k_{1}-k_{2}+m_{1}-m_{2}\right)^{1 / 2}}, \\
& \left|B_{k_{1}, m_{1}, k_{2}, m_{2}}^{(2)}\right| \leq \frac{D_{\alpha, \alpha}}{k_{1}-k_{2}+m_{1}-m_{2}} \sum_{i=-m_{2}+2}^{k_{2}-1} \alpha^{k_{2}-1-i} \leq \frac{H_{\alpha}}{k_{1}-k_{2}+m_{1}-m_{2}} .
\end{aligned}
$$

Obviously, if $m_{1}=m_{2}$ then

$$
B_{k_{1}, m_{1}, k_{2}, m_{2}}^{(1)}=0 \quad \text { and } \quad B_{k_{1}, m_{1}, k_{2}, m_{2}}^{(2)}=\sum_{i=-m_{1}+2}^{k_{1} \wedge k_{2}-1} \mathrm{P}\left(S_{k_{1}-1-i}^{(\alpha)}=k_{1}-1-i\right) \alpha^{k_{2}-1-i} \leq \frac{\alpha^{\left|k_{1}-k_{2}\right|}}{1-\alpha^{2}} .
$$

In this way, by Lemma 2.1 and Proposition 2.3,

$$
\begin{aligned}
& \sum_{m_{1}=1}^{n} \sum_{m_{2}=1}^{n} \sum_{k_{1}=-m_{1}+1}^{m_{1}} \sum_{k_{2}=-m_{2}+1}^{m_{2}}\left|G_{k_{1}, m_{1}, k_{2}, m_{2}}^{(2)}\right| \leq \frac{C_{\alpha}}{1-\alpha^{2}} \sum_{m=1}^{n} \sum_{k_{1}=-m+3}^{m} \sum_{k_{2}=-m+3}^{m}\left(k_{1}+k_{2}+2 m\right)^{1 / 2} \alpha^{\left|k_{1}-k_{2}\right|} \\
& \quad+H_{\alpha} \sum_{m_{1}=2}^{n} \sum_{m_{2}=1}^{m_{1}-1} \sum_{k_{2}=-m_{2}+1}^{m_{2}} \sum_{k_{1}=-m_{1}+3}^{k_{2}-1} \alpha^{k_{2}-k_{1}}\left(k_{2}+m_{1}\right)^{1 / 2}\left(K_{\alpha}+2 C_{\alpha} \log \left(k_{2}+m_{1}\right)\right) \\
& \quad+H_{\alpha} \sum_{m_{1}=2}^{n} \sum_{m_{2}=1}^{m_{1}-1} \sum_{k_{1}=-m_{1}+3}^{m_{1}} \sum_{k_{2}=-m_{2}+1}^{k_{1}}\left(\frac{K_{\alpha}}{\left(k_{1}-k_{2}+m_{1}-m_{2}\right)^{1 / 2}}+\frac{C_{\alpha}\left(k_{1}+k_{2}+m_{1}+m_{2}\right)^{1 / 2}}{k_{1}-k_{2}+m_{1}-m_{2}}\right) \\
& \leq \\
& \quad \frac{8 C_{\alpha}}{(1-\alpha)\left(1-\alpha^{2}\right)} \sum_{m=1}^{n} m^{3 / 2}+\frac{H_{\alpha}}{1-\alpha} \sum_{m=2}^{n} \sum_{k=-m+1}^{m} m(k+m)^{1 / 2}\left(K_{\alpha}+2 C_{\alpha} \log (k+m)\right) \\
& \quad+2 H_{\alpha} \sum_{m=2}^{n} \sum_{k=-m+3}^{m} m(k+m)^{1 / 2}\left(K_{\alpha}+2 C_{\alpha} \log (k+m)\right) \leq Q_{\alpha}(n+1)^{7 / 2} \log (n+1)
\end{aligned}
$$

with some constant $Q_{\alpha}>0$. Inequalities (4.7) and (4.8) imply

$$
\begin{aligned}
\sum_{m_{1}=1}^{n} \sum_{m_{2}=1}^{n} \sum_{k_{1}=-m_{1}+1}^{m_{1}} & \sum_{k_{2}=-m_{2}+1}^{m_{2}} G_{m_{1}, m_{2}, k_{1}, m_{1}, k_{2}, m_{2}} \\
= & \sum_{m_{1}=1}^{n} \sum_{m_{2}=1}^{n} \sum_{k_{1}=-m_{1}+1}^{m_{1}} \sum_{k_{2}=-m_{2}+1}^{m_{2}} G_{k_{1}, m_{1}, k_{2}, m_{2}}^{(1)}+\mathcal{O}\left(n^{7 / 2} \log (n)\right),
\end{aligned}
$$

and the same can be proved for the remaining three terms of (4.6). Hence

$$
\begin{aligned}
\operatorname{Var}\left(\sum_{m=1}^{n} U_{m}\right)=\sum_{m_{1}=1}^{n} \sum_{\left(k_{1}, \ell_{1}\right) \in T_{m_{1} \backslash} \backslash T_{m_{1}-1}} \sum_{m_{2}=1}^{n} \sum_{\left(k_{2}, \ell_{2}\right) \in T_{m_{2}} \backslash T_{m_{2}-1}} G_{k_{1}, \ell_{1}, k_{2}, \ell_{2}}^{(1)} & \sum_{\left(k_{1}, \ell_{1}\right) \in T_{n}} \sum_{\left(k_{2}, \ell_{2}\right) \in T_{n}} G_{k_{1}, \ell_{1}, k_{2}, \ell_{2}}^{(1)}+\mathcal{R}_{n},
\end{aligned}
$$

and $\mathcal{R}_{n}=\mathcal{O}\left(n^{7 / 2} \log (n)\right)$. Further, Lemma (2.4) implies

$$
G_{k_{1}, \ell_{1}, k_{2}, \ell_{2}}^{(1)}=\mathcal{L}_{k_{1}, \ell_{1}, k_{2}, \ell_{2}}^{(1)}+\mathcal{L}_{k_{1}, \ell_{1}, k_{2}, \ell_{2}}^{(2)}
$$


where

$$
\begin{aligned}
\mathcal{L}_{k_{1}, m_{1}, k_{2}, m_{2}}^{(1)}:= & \operatorname{Cov}\left(X_{k_{1}-1, m_{1}}-X_{k_{1}, m_{1}-1}, X_{k_{2}-1, m_{2}}-X_{k_{2}, m_{2}-1}\right) \operatorname{Cov}\left(X_{k_{1}, m_{1}-1}, X_{k_{2}, m_{2}-1}\right) \\
& +\operatorname{Cov}\left(X_{k_{1}-1, m_{1}}-X_{k_{1}, m_{1}-1}, X_{k_{2}, m_{2}-1}\right) \operatorname{Cov}\left(X_{k_{2}-1, m_{2}}-X_{k_{2}, m_{2}-1}, X_{k_{1}, m_{1}-1}\right)
\end{aligned}
$$

and using the same ideas as in the proof of (3.4) on can show

$$
\lim _{n \rightarrow \infty} n^{-9 / 2} \sum_{\left(k_{1}, \ell_{1}\right) \in T_{n}} \sum_{\left(k_{2}, \ell_{2}\right) \in T_{n}} \mathcal{L}_{k_{1}, \ell_{1}, k_{2}, \ell_{2}}^{(2)}=0 .
$$

Finally,

$$
\begin{aligned}
n^{-9 / 2} \sum_{\left(k_{1}, \ell_{1}\right) \in T_{n}} & \sum_{\left(k_{2}, \ell_{2}\right) \in T_{n}} \mathcal{L}_{k_{1}, \ell_{1}, k_{2}, \ell_{2}}^{(1)} \\
=\iiint \int_{T}( & \sqrt{n} \operatorname{Cov}\left(Z_{0,1}^{(n)}\left(s_{1}, t_{1}\right)-Z_{1,0}^{(n)}\left(s_{1}, t_{1}\right), Z_{0,1}^{(n)}\left(s_{2}, t_{2}\right)-Z_{1,0}^{(n)}\left(s_{2}, t_{2}\right)\right) \\
& \times \operatorname{Cov}\left(Z_{1,0}^{(n)}\left(s_{1}, t_{1}\right), Z_{1,0}^{(n)}\left(s_{2}, t_{2}\right)\right)+\operatorname{Cov}\left(Z_{0,1}^{(n)}\left(s_{1}, t_{1}\right)-Z_{1,0}^{(n)}\left(s_{1}, t_{1}\right), Z_{1,0}^{(n)}\left(s_{2}, t_{2}\right)\right) \\
& \left.\times \sqrt{n} \operatorname{Cov}\left(Z_{0,1}^{(n)}\left(s_{2}, t_{2}\right)-Z_{1,0}^{(n)}\left(s_{2}, t_{2}\right), Z_{1,0}^{(n)}\left(s_{1}, t_{1}\right)\right)\right) \mathrm{d} s_{1} \mathrm{~d} t_{1} \mathrm{~d} s_{2} \mathrm{~d} t_{2} .
\end{aligned}
$$

With the help of Lemma 2.1 and Proposition 2.3 one can easily show that the integrand on the right hand side of (4.11) can be dominated by $K_{\alpha}\left(C_{\alpha} \sqrt{s_{1}+t_{1}+s_{2}+t_{2}+1}+K_{\alpha}\right)$, which has a finite integral on $T \times T$. Hence, by Proposition 2.2 and dominated convergence theorem

$$
\lim _{n \rightarrow \infty} n^{-9 / 2} \sum_{\left(k_{1}, \ell_{1}\right) \in T_{n}} \sum_{\left(k_{2}, \ell_{2}\right) \in T_{n}} \mathcal{L}_{k_{1}, \ell_{1}, k_{2}, \ell_{2}}^{(1)}=0,
$$

which together with (4.9) and (4.10) implies (4.5).

Proof of Proposition 4.2. We have

$$
\mathbb{1}_{\left\{\left\|\widetilde{d}_{T_{m}}-\widetilde{d}_{T_{m-1}}\right\| \geq \delta\right\}} \leq \delta^{-2}\left\|\widetilde{d}_{T_{m}}-\widetilde{d}_{T_{m-1}}\right\|^{2},
$$

hence to prove the proposition it suffices to show

$$
\sum_{m=1}^{n} \mathrm{E}\left(\left\|\widetilde{d}_{T_{m}}-\widetilde{d}_{T_{m-1}}\right\|^{4} \mid \mathcal{F}_{m-1}\right) \stackrel{\mathrm{P}}{\longrightarrow} 0 \quad \text { as } n \rightarrow \infty
$$

which is a direct consequence of

$$
n^{-4} \sum_{m=1}^{n} \mathrm{E}\left(\left|d_{m}^{(1)}-d_{m-1}^{(1)}\right|^{4} \mid \mathcal{F}_{m-1}\right) \stackrel{\mathrm{P}}{\longrightarrow} 0 \quad \text { and } \quad n^{-5} \sum_{m=1}^{n} \mathrm{E}\left(\left|d_{m}^{(2)}-d_{m-1}^{(2)}\right|^{4} \mid \mathcal{F}_{m-1}\right) \stackrel{\mathrm{P}}{\longrightarrow} 0 .
$$

However, these statements have already been proved, see Baran et al. (2005, Section 6, pp. 47-48) and Baran et al. (2005, Section 4, pp. 31-32), respectively.

Acknowledgments. Research has been supported by the Hungarian Scientific Research Fund under Grants No. OTKA T079128/2009 and OTKA NK101680. G. Pap has been partially supported by the TÁMOP-4.2.2.A-11/1/KONV project. 


\section{References}

Baran, S., Pap, G. (2011) Parameter estimation in a spatial unit root autoregressive model. J. Multivariate Anal. 107, 282-305.

Baran, S., Pap, G. and Zuijlen, M. v. (2004) Asymptotic inference for an unstable spatial AR model. Statistics 38, 465-482.

Baran, S., Pap, G. and Zuijlen, M. v. (2005) Asymptotic inference for unit roots in spatial triangular autoregression. Department of Mathematics, Radboud University Nijmegen, The

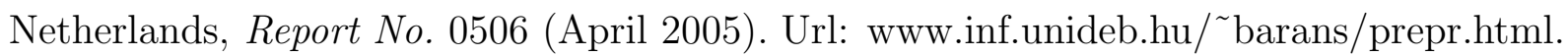

Baran, S., Pap, G. and Zuijlen, M. v. (2007) Asymptotic inference for unit roots in spatial triangular autoregression. Acta Appl. Math. 96, 17-42.

Basu, S. and Reinsel, G. C. (1993) Properties of the spatial unilateral first-order ARMA model. Adv. in Appl. Probab. 25, 631-648.

Besag, J. E. (1972) On the correlation structure of some two dimensional stationary processes. Biometrika 59, 43-48.

Hamilton, J. D. (1994) Time Series Analysis. Princeton University Press, Princeton.

Jacod, J. and Shiryaev, A. N. (1987) Limit Theorems for Stochastic Processes. SpringerVerlag, Berlin.

Paulauskas, V. (2007) On unit roots for spatial autoregressive models. J. Multivariate Anal. 98, 209-226.

Sims, J. D., Stock, J. H. and Watson, M. W. (1990) Inference in linear time series models with some unit roots. Econometrica 58, 113-144.

Whittle, P. (1954) On stationary processes in the plane. Biometrika 41, 434-449. 